\title{
Monocyte/macrophage androgen receptor suppresses cutaneous wound healing in mice by enhancing local TNF- $\alpha$ expression
}

\author{
Jiann-Jyh Lai, Kuo-Pao Lai, Kuang-Hsiang Chuang, Philip Chang, \\ I-Chen Yu, Wen-Jye Lin, and Chawnshang Chang
}

George Whipple Lab for Cancer Research, Department of Pathology, Department of Urology, Department of Radiation Oncology, and Wilmot Cancer Center, University of Rochester Medical Center, Rochester, New York, USA.

\begin{abstract}
Cutaneous wounds heal more slowly in elderly males than in elderly females, suggesting a role for sex hormones in the healing process. Indeed, androgen/androgen receptor (AR) signaling has been shown to inhibit cutaneous wound healing. AR is expressed in several cell types in healing skin, including keratinocytes, dermal fibroblasts, and infiltrating macrophages, but the exact role of androgen/AR signaling in these different cell types remains unclear. To address this question, we generated and studied cutaneous wound healing in cell-specific $A R$ knockout (ARKO) mice. General and myeloid-specific ARKO mice exhibited accelerated wound healing compared with WT mice, whereas keratinocyte- and fibroblast-specific ARKO mice did not. Importantly, the rate of wound healing in the general ARKO mice was dependent on AR and not serum androgen levels. Interestingly, although dispensable for wound closure, keratinocyte AR promoted re-epithelialization, while fibroblast AR suppressed it. Further analysis indicated that AR suppressed wound healing by enhancing the inflammatory response through a localized increase in TNF- $\alpha$ expression. Furthermore, AR enhanced local TNF- $\alpha$ expression via multiple mechanisms, including increasing the inflammatory monocyte population, enhancing monocyte chemotaxis by upregulating CCR2 expression, and enhancing TNF- $\alpha$ expression in macrophages. Finally, targeting AR by topical application of a compound (ASC-J9) that degrades AR protein resulted in accelerated healing, suggesting a potential new therapeutic approach that may lead to better treatment of wound healing.
\end{abstract}

\section{Introduction}

Wound healing is a complicated process composed of several overlapping phases, the inflammatory, proliferative, and remodeling phases. Delayed cutaneous wound healing usually results in local infection and may potentially lead to chronic, nonhealing wounds (1). Clinically, cutaneous wounds heal more slowly in elderly males than in elderly females and are accompanied by increased inflammatory cell infiltration and reduced collagen deposition (1-3). Other studies have also shown that the male gender in the elderly population is a risk factor for impaired wound healing (4). Collectively, these data suggest that sex hormones, including androgens, might play important roles in the healing process.

Testosterone is the major androgen in circulation and is mostly produced by Leydig cells of the testis. Testosterone can be further catalyzed by $5 \alpha$-reductase into $5 \alpha$-dihydrotestosterone (DHT), which is a more potent androgen than testosterone and has a 10-fold higher affinity for androgen receptor (AR) (5). AR is a member of the nuclear receptor superfamily. Upon androgen binding, it becomes activated and translocates into the nucleus to modulate expression of its target genes $(6,7)$. The expression of AR in the healing skin has been detected in keratinocytes, dermal fibroblasts, and infiltrating macrophages, implying a possible role in the healing process (1).

Earlier studies by Ashcroft and colleagues, using surgical or chemical castration, have found that androgens were able to inhibit

Conflict of interest: ASC-J9 was patented by the University of Rochester, the University of North Carolina, and AndroScience Corp., and then licensed to AndroScience Corp. Both the University of Rochester and C. Chang own royalties and equity in AndroScience Corp.

Citation for this article: J. Clin. Invest. doi:10.1172/JCI39335. cutaneous wound healing, possibly by modulating inflammatory responses, matrix deposition, and keratinocyte function $(1,8-10)$. However, the in vivo role of androgens/AR signals in different cell types involved in the wound-healing process remains unclear. In addition, increasing evidence suggests that androgens do not necessarily act through AR (11), while AR also has some androgenindependent functions (12-14). However, the approaches using surgical or chemical castration to diminish androgen levels cannot separate the effects of AR from androgens. Therefore, it is necessary to develop a better in vivo system to more definitively clarify the role of androgens/AR signals in the regulation of wound healing.

In this study, we used cell-specific AR knockout (ARKO) mice (15) and reciprocal bone marrow transplantation to dissect AR function in different cell types involved in the healing skin, and we demonstrate that $\mathrm{AR}$ in macrophages, rather than in keratinocytes and dermal fibroblasts, was critical in the inhibition of cutaneous wound healing. Using in vivo functional studies, we clarified that local TNF- $\alpha$ production from macrophages critically mediated the suppressive effect of androgen/AR in the healing wound. Further in vivo and in vitro mechanistic studies demonstrated that AR could enhance local TNF- $\alpha$ production through multiple mechanisms. Finally, we demonstrate the feasibility of local AR targeting as a potential therapy to accelerate wound healing using topical treatment of ASC-J9, a newly developed anti-AR compound that degrades AR with little influence on the serum testosterone concentration.

\section{Results}

Cutaneous wound bealing is accelerated in mice lacking AR. To understand the AR roles in each cell type involved in wound healing and test the potential therapeutic roles of $\mathrm{AR}$ in wound healing, 
A

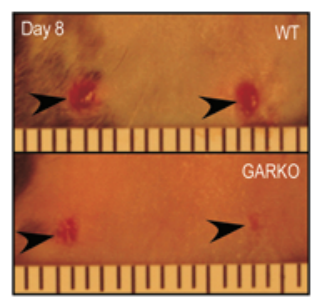

D

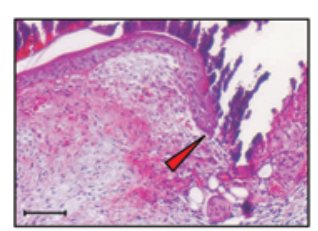

B

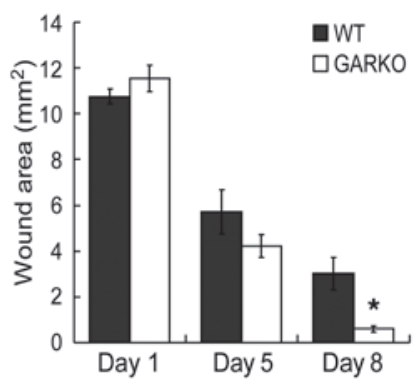

E

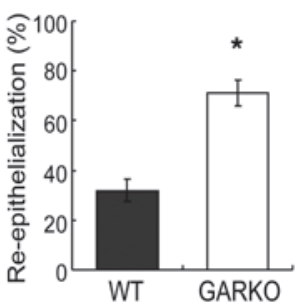

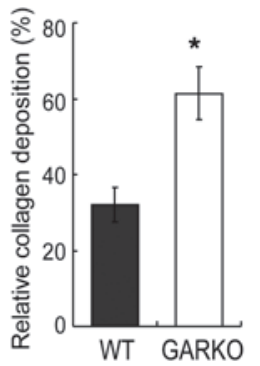

G

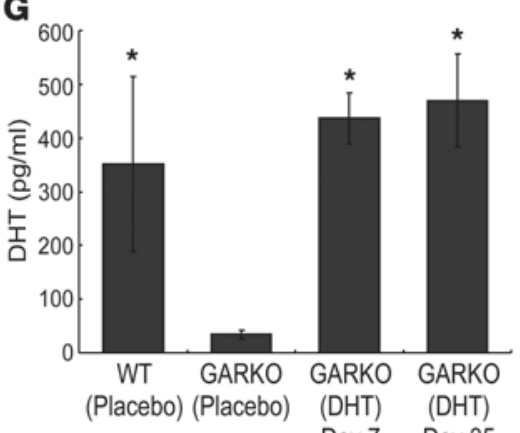

Day 7 Day 35
F

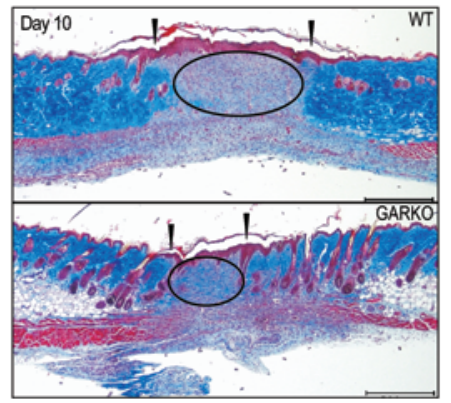

\section{C}

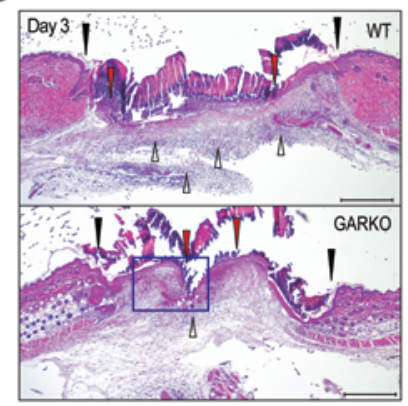

WT (Placebo)

$\square$ GARKO (Placebo)

$\square$ GARKO (DHT)

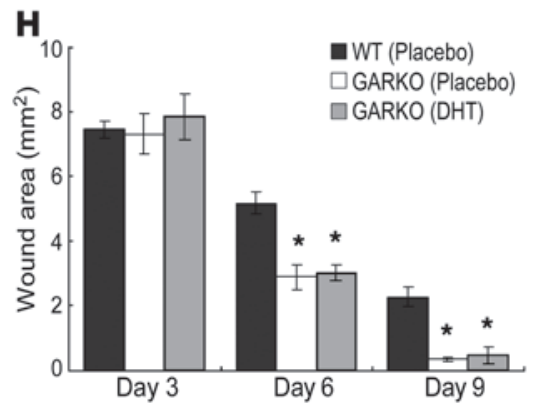

\section{Figure 1}

Cutaneous wound healing is accelerated in male GARKO mice due to loss of AR function. Excisional wounds were made by 4-mm-diameter punches. (A) Photographs of day 8 wounds. The arrowheads point out the location of wounds. (B) Wound areas were quantified for WT and GARKO mice. $n=7-10$ (2 wounds/mouse). ${ }^{*} P=0.001$. (C) H\&E staining for day 3 wound sections. Black arrowheads indicate edges of wounds, red arrowheads indicate edges of epithelium, and white arrowheads indicate foci of infiltrating cells. Scale bars: $500 \mu \mathrm{m}$. (D) Higher magnification of the boxed region in C. The red arrowhead indicates the edge of epithelium. Scale bar: $100 \mu \mathrm{m}$. (E) The quantified data for re-epithelialization. $n=4$ (6 wounds/mouse). ${ }^{\star} P=0.001$. (F) Day 10 wounds were subjected to Mason's Trichrome staining to detect collagen fibers (blue). Arrowheads indicate the edges of wounds, and ovals indicate the areas of granulation tissue. Data are representative of 12-16 wounds (4 wounds/ mouse) in each group. Scale bars: $500 \mu \mathrm{m}$. The quantitative data show relative collagen deposition in granulation tissues compared with the adjacent dermis. $n=3-4$ (4 wounds/mouse). ${ }^{\star} P<0.05$. (G) DHT pellets or placebos were implanted into GARKO mice, and only placebo pellets were implanted into WT mice. Serum DHT levels were detected by ELISA after DHT restoration. $n=3-4$ mice. ${ }^{*} P=0.023-0.025$ versus GARKO placebo. (H) After DHT restoration, wounds were created, and wound areas were quantified on the days indicated. $n=3-4$ (2 wounds $/$ mouse). ${ }^{\star} P<0.01$ versus WT (placebo).

we first generated the general ARKO (GARKO) mice by breeding fAR mice (carrying loxP-flanked AR allele) (15) with ACTBCre mice (carrying cre transgene driven by $\beta$-actin promoter) (Supplemental Figure 1A; supplemental material available online with this article; doi:10.1172/JCI39335DS1). Excision wounds were then made on the dorsal skin of male GARKO mice and their WT littermates. Interestingly, we found that the cutaneous wounds on GARKO mice healed faster than those on the WT mice, suggesting that AR suppresses wound healing (Figure 1, A and B). Histological comparison of day 3 wounds revealed that re-epi- thelialization in GARKO mice, an early indicator of wound healing (16), was accelerated compared with that in WT mice (Figure $1, \mathrm{C}-\mathrm{E})$. Trichrome staining in day 10 wounds was increased in GARKO granulation tissues, indicating that collagen deposition was enhanced in GARKO versus WT wounds (Figure 1F). Collectively, these data suggest that AR represses collagen deposition, epithelium regrowth, and overall wound healing.

Because serum testosterone levels are significantly reduced in the GARKO compared with WT mice (15) and studies have shown that androgens can act through AR-independent pathways to reg- 


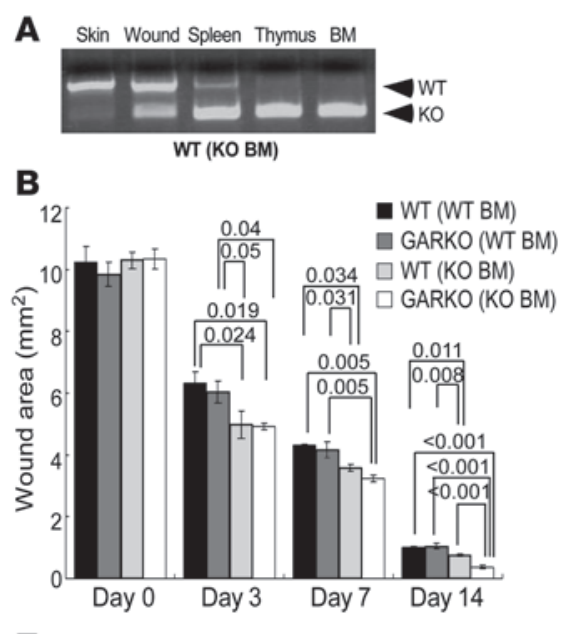

E

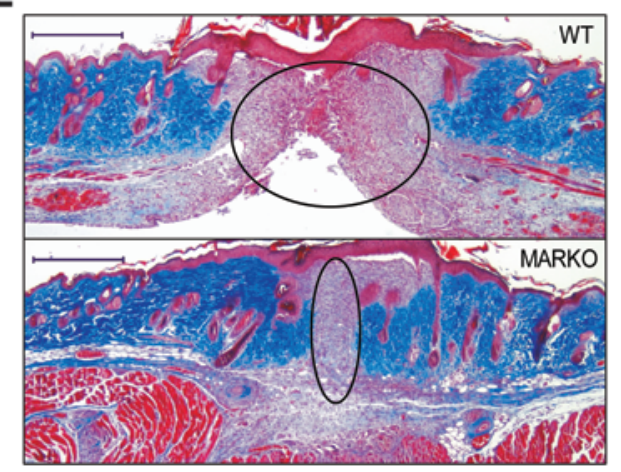

G

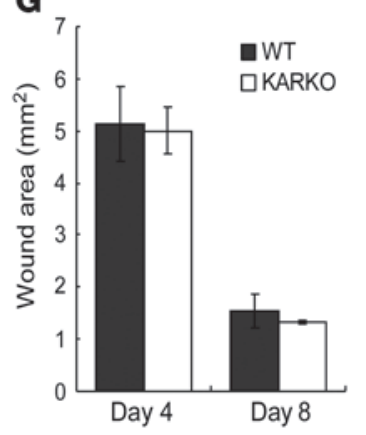

C BM Skin D3W Spln Thy Lung Liver Mus Tes Bld

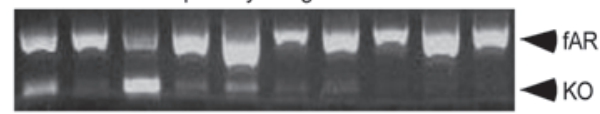

D
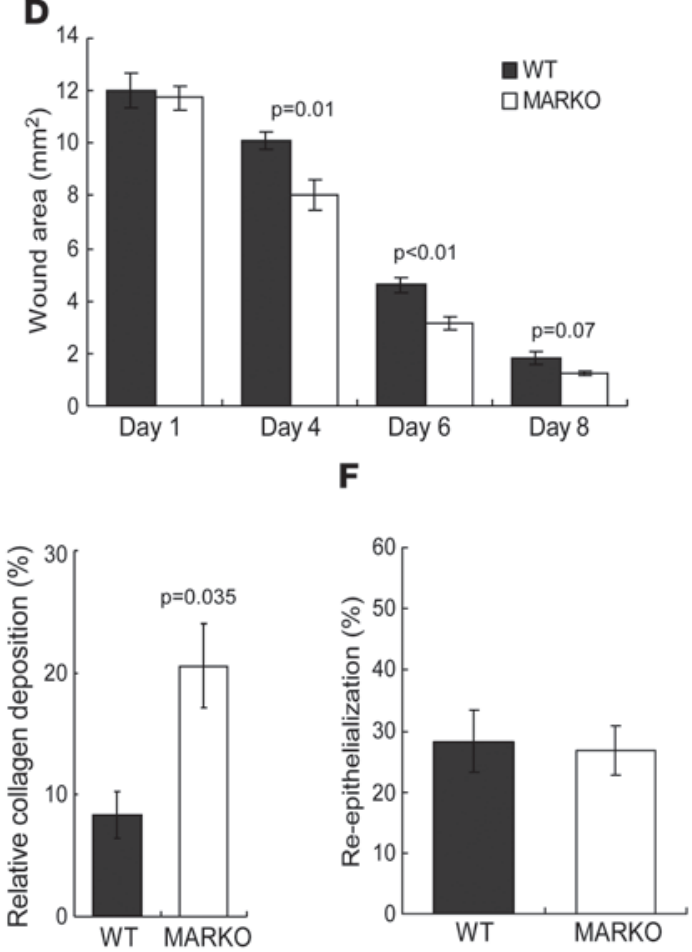

H

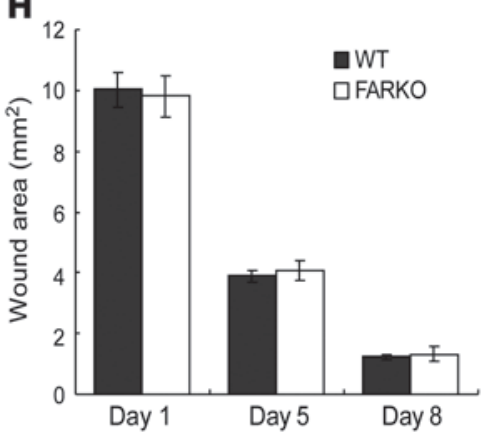

Figure 2

AR in monocytes/macrophages is critical for suppressing wound healing. (A) $\gamma$-Irradiated 6-week-old WT and GARKO mice were adoptively transplanted with WT or GARKO bone marrow cells. To confirm the bone marrow engraftment, various tissues from a WT recipient receiving GARKO bone marrow were subjected to AR genotyping. KO, truncated AR allele. (B) After bone marrow engraftment, wounds were created and the wound areas were quantified and compared among recipients. $n=3-4$ (6 wounds/mouse). The numbers indicate the $P$ values for each comparison. (C) Various tissues from MARKO mice were subjected to AR genotyping to confirm the efficiency and specificity of AR deletion. D3W, day 3 wound; Thy, thymus; Spln, spleen; Mus, muscle; Tes, testis; Bld, bladder. (D) Wound areas were compared between male MARKO mice and their WT littermates. $n=6-7$ (2 wounds/mouse). (E) Day 8 wound tissues from WT and MARKO mice were subjected to Trichrome staining to detect collagen fibers (blue). Ovals indicate granulation tissues. Scale bars: $500 \mu \mathrm{m}$. The quantitative data show relative collagen deposition in granulation tissues compared with the adjacent dermis. $n=3$ (4 wounds/mouse). (F) Day 3 wound tissues from WT and MARKO mice were subjected to H\&E staining for analysis of re-epithelialization. $n=4$ ( 6 wounds/mouse). (G) Excisional wounds were created and compared between KARKO mice and their WT littermates. $n=3-4$ (6 wounds/mouse). (H) Excisional wounds were created and compared between FARKO mice and their WT littermates. $n=4-6$ (6 wounds/mouse). Due to differences in genetic backgrounds among conditional ARKO lines, the WT controls among different experiments were not necessarily equivalent.

ulate cellular activities $(11,17,18)$, we therefore asked whether the accelerated wound-healing phenotype observed in GARKO mice was due to AR deficiency or reduced serum androgens. To address this question, we subcutaneously implanted DHT pellets into
GARKO mice to restore their serum androgen levels (Figure $1 \mathrm{G}$ ) and found that DHT restoration failed to reverse the acceleration of wound healing (Figure 1H). This suggests that the accelerated wound-healing phenotype observed in GARKO mice is not due 

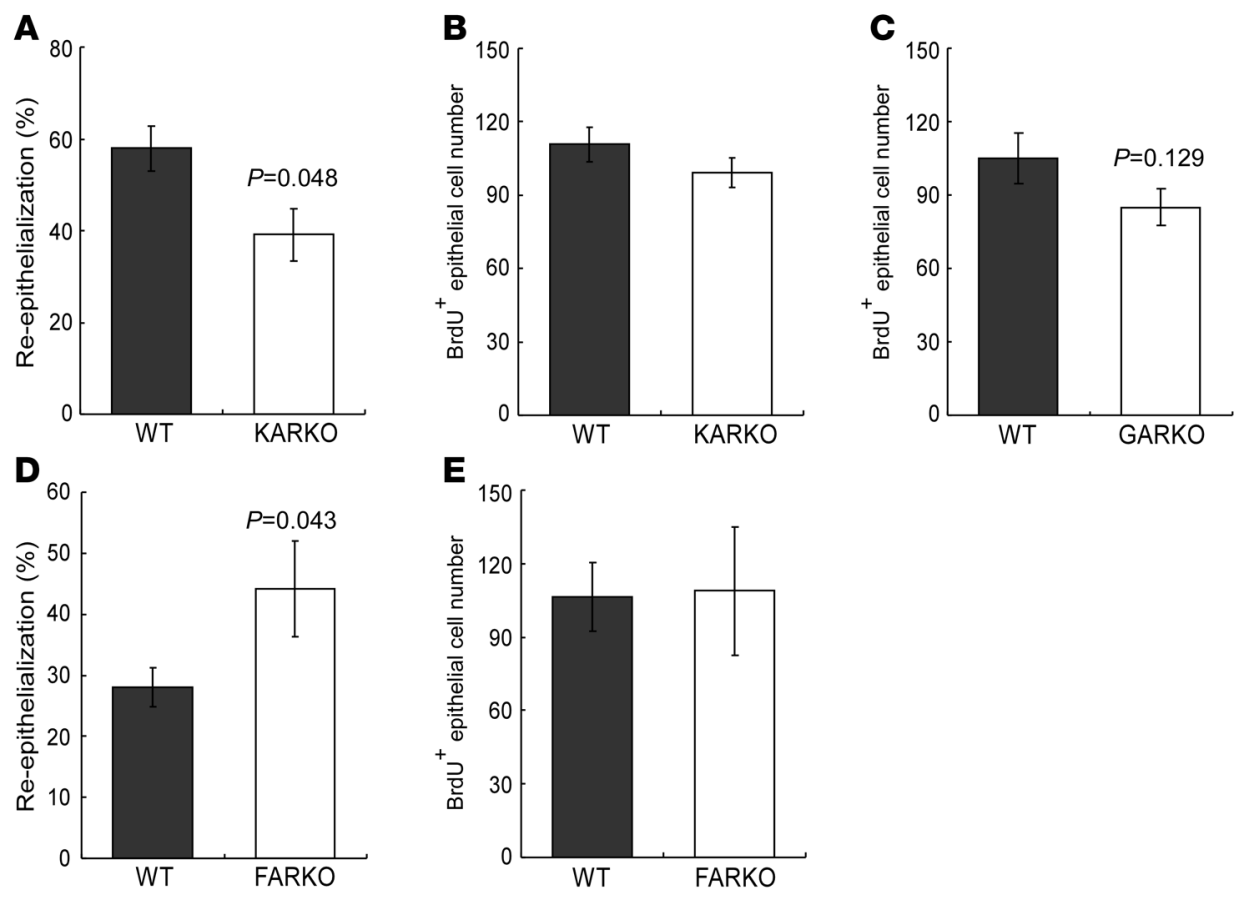

Figure 3

AR in keratinocytes and fibroblasts is involved in regulating re-epithelialization. (A) Day 4 wounds from KARKO and WT mice were subjected to paraffin sectioning and H\&E staining to determine the re-epithelialization rate. $n=4-6$ (6 wounds/mouse). (B and C) Day 3 wounds were harvested from mice that had been injected with BrdU (150 $\mu \mathrm{g} / \mathrm{g}$ body weight) 2 hours before harvest. The wounds were subjected to paraffin sectioning and subsequent immunohistochemistry to detect BrdU incorporation. $\mathrm{BrdU}^{+}$epithelial cells in each wound section of (B) KARKO and (C) GARKO mice and their WT littermates were counted. $n=5-6$ ( 6 wounds/mouse). (D) Re-epithelialization rates of day 3 wounds were compared between FARKO and WT mice. $n=4-6$ (6 wounds/mouse). (E) Day 3 wounds were harvested from FARKO and WT mice injected with BrdU and subjected to immunohistochemistry as described in $\mathbf{B}$ and $\mathbf{C}$. The proliferating $\left(\mathrm{BrdU}^{+}\right)$epithelial cells were counted in each wound section. $n=4-6$ (4 wounds/mouse).

to reduced serum androgen levels, and AR-dependent pathways are critical, whereas AR-independent pathways are dispensable in mediating the ability of androgens to suppress wound healing.

Wound healing is accelerated in mice lacking AR in infiltrating inflammatory cells. The healing skin has 2 major cell compartments: resident skin cells, which include keratinocytes and dermal fibroblasts, and infiltrating cells, which include inflammatory cells (19). To investigate the relative role of AR in each cell compartment, we adoptively transplanted GARKO or WT bone marrow cells into $\gamma$-irradiated WT or GARKO mice to replace the hematopoietic system, and hence the inflammatory cell compartment, of recipient mice. Genotyping results from various tissues in recipient mice demonstrated that the majority of the Ar gene in the non-wounded skin was of the recipient genotype (WT allele, in this case), while the donor genotype (KO allele) predominated in the wounded skin due to massive inflammatory cell infiltration into the wound (Figure $2 \mathrm{~A}$ ). As expected, the majority of the Ar gene in the spleen, thymus, and bone marrow was of the donor genotype, indicating that bone marrow transplantation had successfully replaced the hematopoietic system of recipient mice.

Upon comparison of the wound-healing rates among the 4 groups of recipient mice, we found that WT mice reconstituted with WT bone marrow had the slowest wound-healing rate, while
GARKO mice reconstituted with GARKO bone marrow had the fastest healing rate (Figure $2 \mathrm{~B}$ ). Interestingly, when GARKO mice were transplanted with WT bone marrow, the healing rate was delayed to rates comparable to that of WT mice transplanted with WT bone marrow, although serum androgen levels in GARKO recipients remained low. This suggests that $\mathrm{AR}$ in the infiltrating inflammatory cell compartment may function in an androgen-independent manner to suppress wound healing. In contrast, WT mice reconstituted with GARKO bone marrow had accelerated wound healing compared with WT mice reconstituted with WT bone marrow, despite comparable serum androgen levels (Figure 2B). Taken together, the results from these reciprocal bone marrow transplantations clearly demonstrate that AR in the infiltrating inflammatory cell compartment, but not in the resident skin cells, plays a critical role in suppressing wound healing.

Wound bealing is accelerated in myeloid-specific ARKO mice. Next, we asked in which population of infiltrating inflammatory cells AR played an essential role in suppressing wound healing. Among woundinfiltrating cells, macrophages are known to be central regulators for the healing process $(20,21)$. Therefore, we crossed fAR mice with LyzCre mice (22) to generate myeloid-specific ARKO mice (MARKO mice). Even though AR in granulocytes was also deleted in MARKO mice, granulocytes, especially neutrophils, are not important for regulating wound healing in the absence of infections $(23,24)$, as was the case in our system. Thus MARKO mice appear to be a good model to study the role of monocyte/ macrophage AR in the regulation of wound healing. Genotyping confirmed that AR was selectively deleted in MARKO mice, with the knockout allele most abundant in the bone marrow and day 3 wounds. A lesser amount of knockout allele was detected in the normal skin, spleen, thymus, lung, and liver, whereas in other tissues, the knockout allele was barely detected (Figure 2C). Interestingly, MARKO mice exhibited accelerated wound healing compared with WT mice (Figure 2D) and had increased collagen deposition in the later stages of wound healing (Figure 2E). However, re-epithelialization was comparable between MARKO and WT wounds (Figure 2F), suggesting that AR in the monocytes/ macrophages is critical for repressing overall wound healing and collagen deposition but is not essential in regulating re-epithelialization. In contrast, the overall healing rates were comparable between keratinocyte-specific ARKO mice (KARKO mice, which were generated by mating fAR with K5Cre mice (25); Supplemen- 

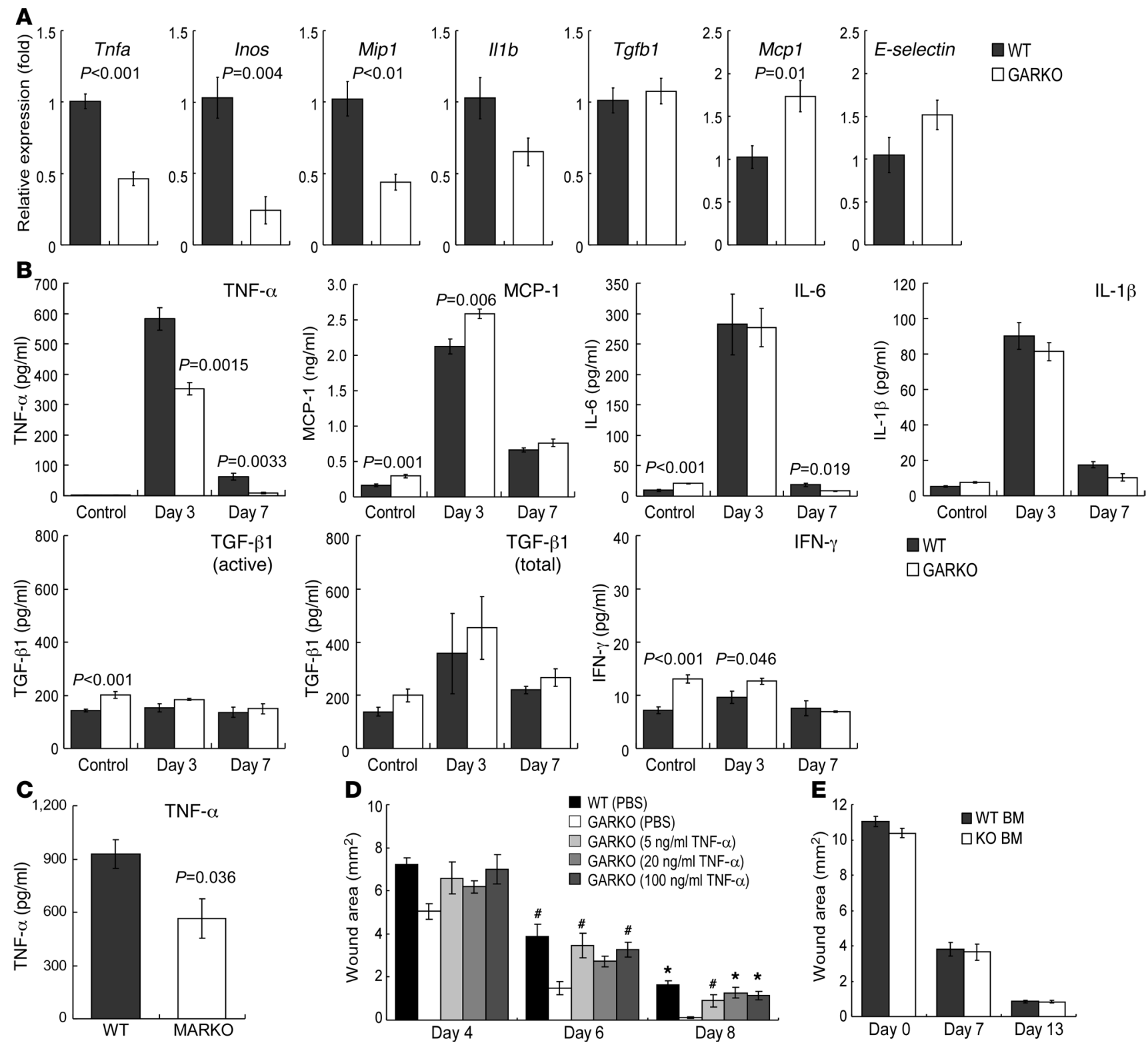

Figure 4

TNF- $\alpha$ is critical for mediating the ability of AR to suppress wound healing. (A) Relative mRNA expression of various inflammatory mediators was measured by quantitative RT-PCR in day 3 wound tissues of WT and GARKO mice. $n=4-8$. (B) Proteins were extracted from the wounded (day 3 and day 7) or non-wounded skin (control) and subjected to ELISA to detect the expression of inflammatory mediators. The concentration of TGF- $\beta 1$ was measured without (active) or with (total) acid activation. $n=4-7$. (C) TNF- $\alpha$ expression was detected by ELISA in day 3 wound tissues of MARKO mice and their WT littermates. $n=4$. (D) One day after creating wounds, various concentrations of TNF- $\alpha$ were intradermally injected around GARKO wounds, while WT wounds were injected with PBS. The wound areas were quantified on days 4,6 , and 8. $n=3-4$ (2 wounds/mouse). $\# P<0.05,{ }^{*} P<0.01$ versus GARKO (PBS) group. (E) $\gamma$-Irradiated 6-week-old TNF-R1KO recipient mice were transplanted with bone marrow cells from WT or GARKO mice. After engraftment, wounds were created and the wound areas were quantified. $n=3-4$ (6 wounds/mouse).

tal Figure 1C) or fibroblast-specific ARKO mice (FARKO mice, which were generated by mating fAR with Fsp1Cre mice; ref. 26; Supplemental Figure 1D) and their WT littermates (Figure 2, G and $\mathrm{H}$ ), highlighting the essential role of AR in monocytes/macrophages for wound-healing suppression. It is noteworthy that the genetic backgrounds of the different conditional ARKO lines were different and thus the WT controls of different lines were not necessarily equivalent. However, GARKO mice showed accelerated healing under both C57BL/ 6 and C57BL/ $6 \times$ FVB F1 backgrounds (data not shown), suggesting that the genetic background might not influence the regulatory effects of AR on wound healing.

$A R$ in keratinocytes and fibroblasts is involved in the regulation of reepithelialization. Even though monocyte/macrophage AR is critical in suppressing overall wound healing, MARKO mice did not 

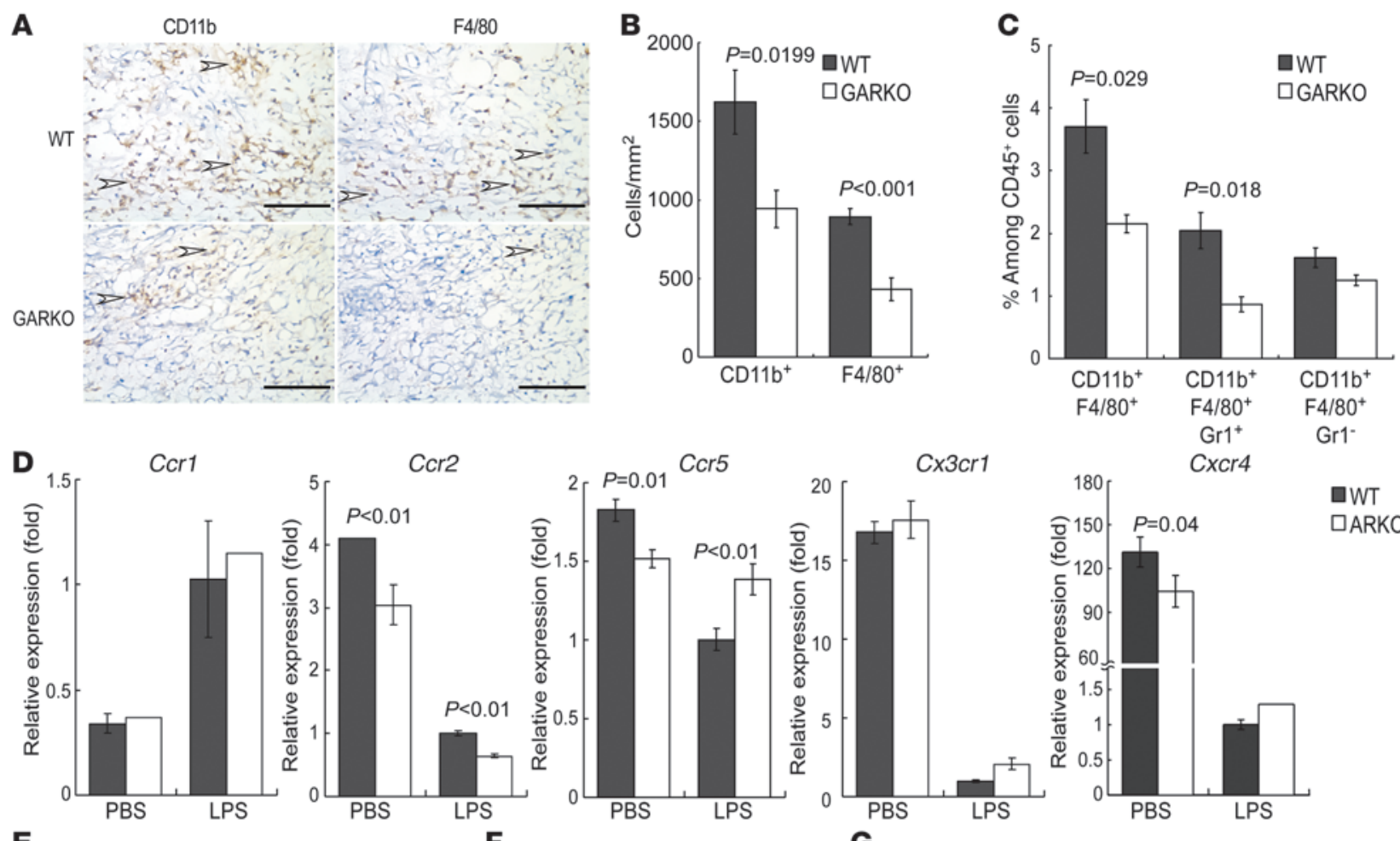

E
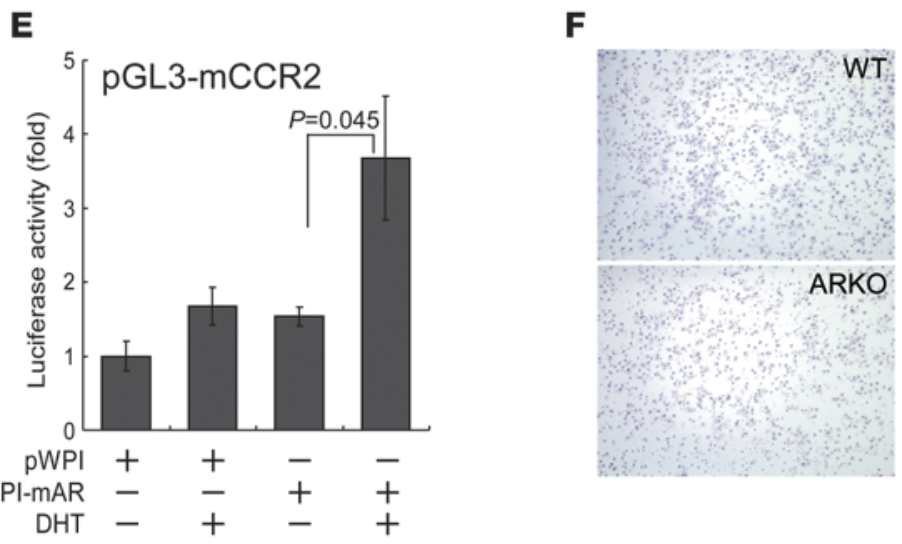

G
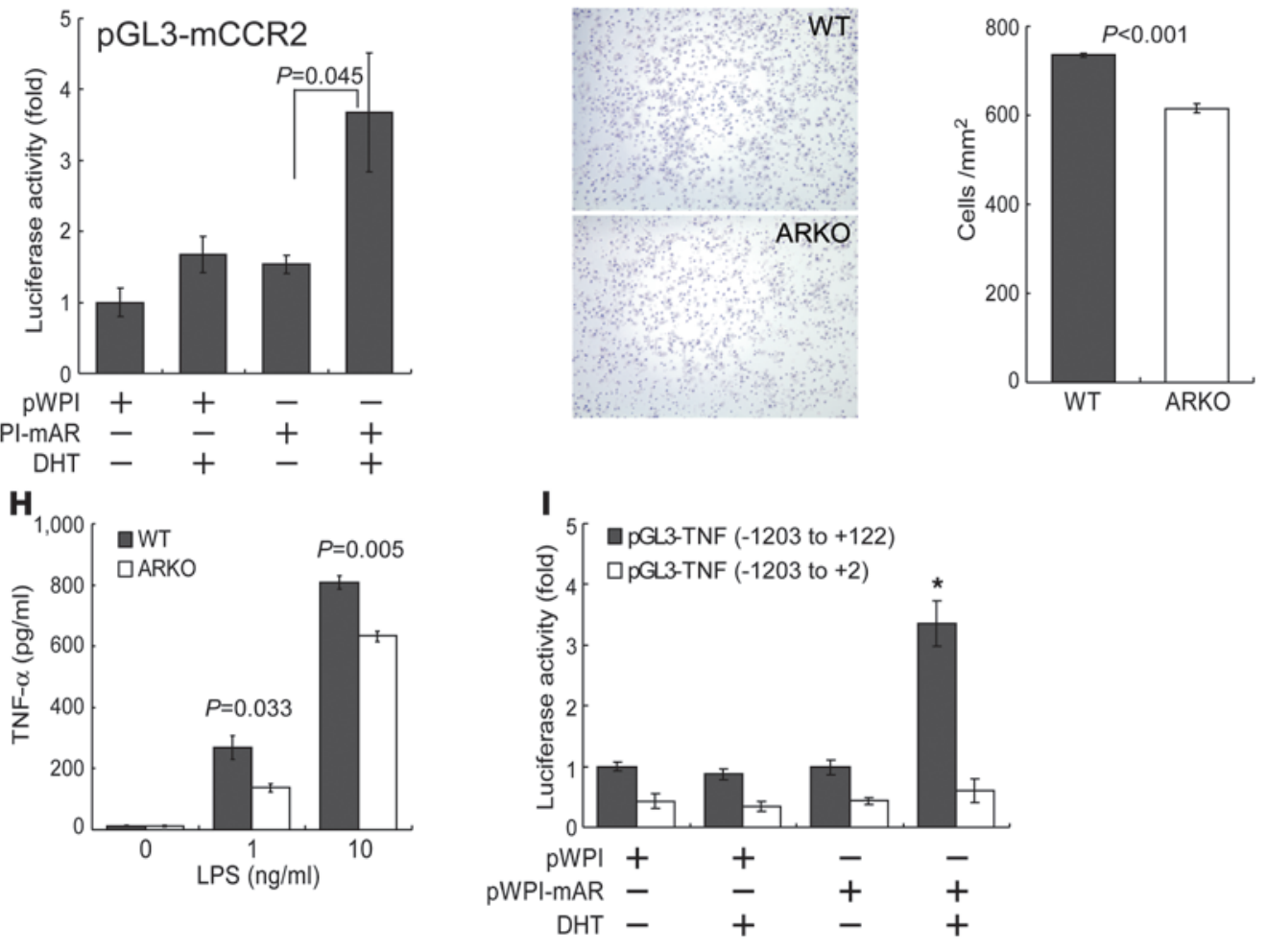


\section{Figure 5}

AR enhances local TNF- $\alpha$ expression through multiple mechanisms. (A) Immunohistochemistry to identify infiltrating myeloid cells (CD11 $\left.\mathrm{b}^{+}\right)$ and macrophages $\left(\mathrm{F} 4 / 80^{+}\right)$in day 3 wounds. White arrows indicate foci of positive-staining cells (brown). Scale bars: $100 \mu \mathrm{m}$. (B) The positivestaining cells were counted in the wound areas. $n=6-7$. (C) Flow cytometry to identify the monocytes $\left(\mathrm{CD} 11 \mathrm{~b}+\mathrm{F} 4 / 80^{+}\right)$, inflammatory monocytes $\left(\mathrm{CD} 11 \mathrm{~b}+\mathrm{F} 4 / 80^{+} \mathrm{Gr} 1^{+}\right)$, and resident monocytes (CD11b+F4/80+Gr1-) in PBMCs. $n=4-9$. (D) mRNA isolated from BMMacs was subjected to quantitative RT-PCR to detect expression of chemokine receptors. For each chemokine receptor, the expression level of WT BMMacs treated with LPS was set as 1. (E) NIH3T3 cells were transiently transfected with reporter containing the promoter region $(-1,258$ to +27$)$ of murine CCR2 together with pWPI-mAR or empty vector. After treating cells with or without DHT, luciferase activities were measured. ( $\mathbf{F}$ and $\mathbf{G}$ ) Transwell chemotaxis assay was used to compare chemotaxis of day 3 BMMacs in response to MCP-1. (F) Images of cells that had migrated across the membrane. Original magnification, $\times 100$. (G) Cell numbers were counted. $n=9$. (H) Day 6 BMMacs were treated with LPS as indicated. TNF- $\alpha$ production was measured by ELISA. (I) Reporters composed of TNF- $\alpha$ promoter containing $5^{\prime}$-UTR (pGL3-TNF, -1,203 to +122 ) or without $5^{\prime}$-UTR ( $p$ GL3-TNF, $-1,203$ to +2 ) were cotransfected into NIH3T3 cells with pWPI-mAR or pWPI. After treating with $\mathrm{DHT}$, luciferase activities were measured. Data in I are mean \pm SD. ${ }^{\star} P<0.001$ versus all other groups.

show all aspects of the wound-healing phenotype of GARKO mice, such as accelerated re-epithelialization, suggesting that AR in other cell types is likely to be involved in the regulation of wound healing. Keratinocytes and dermal fibroblasts are the major structural cells to reconstruct injured skin, although AR in these cells is not essential for regulating overall wound healing. Upon further histological analysis of KARKO wounds, we were surprised to find that the re-epithelialization in KARKO mice was delayed compared with that of WT mice (Figure 3A). However, the numbers of proliferating epithelial cells in the wound areas were similar between KARKO and WT mice (Figure $3 \mathrm{~B}$ ), suggesting that $\mathrm{AR}$ in keratinocytes might actually promote re-epithelialization without influencing keratinocyte proliferation. Interestingly, we also found similar numbers of proliferating epithelial cells in the wounds of GARKO and WT mice (Figure 3C), although the re-epithelialization was accelerated in GARKO wounds (Figure 1E). Because the re-epithelialization rate is determined by both proliferation and migration of keratinocytes $(27,28)$, these data suggest that AR might control re-epithelialization by regulating the migration of keratinocytes rather than their proliferation.

Histological analysis of day 3 wounds in FARKO mice revealed accelerated re-epithelialization compared with that in WT mice (Figure 3D). This suggests that AR in fibroblasts but not myeloid cells or keratinocytes is critical in suppressing re-epithelialization. This suppressive effect is not due to keratinocyte proliferation, as the numbers of proliferating epithelial cells in day 3 wounds were comparable between FARKO and WT mice (Figure 3E). Trichrome staining of day 10 wounds showed similar levels of collagen deposition in granulation tissues of FARKO and WT mice (Supplemental Figure 2), suggesting that AR in fibroblasts is not essential for regulating collagen deposition.

Overall, the data using KARKO and FARKO mice demonstrate that although $\mathrm{AR}$ in keratinocytes and fibroblasts is not essential in suppressing overall wound healing, AR in these 2 cell types plays opposing roles in re-epithelialization.
TNF- $\alpha$ is critical in mediating the suppressive effects of AR on wound healing. Using MARKO mice, we have demonstrated that AR in monocytes/macrophages is critical in suppressing overall wound healing. We hypothesized that AR might suppress wound healing by regulating the inflammatory response, so we examined the expression of key inflammatory mediators (29). Compared with WT wounds, we found that mRNA expression of Tnfa, Mip1, and Inos was significantly decreased in the day 3 GARKO wounds, while Mcp1 expression was increased and Il1 $\beta$, E-selectin, and Tgfb1 expression was not changed significantly (Figure 4A). ELISA analysis confirmed the mRNA data, showing decreased protein expression of TNF- $\alpha$, increased expression of MCP- 1 , and no significant change in TGF- $\beta 1$ and IL- 6 expression in the day 3 wound tissues of GARKO compared with that in WT mice (Figure 4B). Although IFN- $\gamma$ expression was higher in control skins and day 3 wounds of GARKO versus WT mice, the concentrations were very low and were not further induced after wounding, suggesting that the difference in macrophage activation between WT and GARKO mice was not due to a difference in IFN- $\gamma$ levels (Figure 4B).

The expression pattern of inflammatory mediators suggests that AR might enhance the inflammatory response within wound tissues. Among these AR-modulated mediators, we chose to focus on AR-induced local TNF- $\alpha$ expression, based on earlier reports showing that TNF- $\alpha$ plays a critical role in wound healing $(1,30$, 31 , that expression of TNF- $\alpha$ concurrently decreases with castration and accelerated wound healing (1), and that the wound-healing phenotype of TNF receptor-1 knockout (TNF-R1KO) mice (32) markedly parallels that of GARKO mice. Hence, we hypothesized that AR might suppress wound healing by increasing TNF- $\alpha$ expression. We first confirmed that the reduced TNF- $\alpha$ expression seen in GARKO versus WT wounds could be reproduced in MARKO wounds (Figure 4C), and IL-6 was reduced as well in day 3 wounds of MARKO mice (Supplemental Figure 3). Local restoration of TNF- $\alpha$ around GARKO wounds reversed the accelerated healing of GARKO wounds in a dose-dependent fashion (Figure 4D), suggesting that an AR-mediated increase in TNF- $\alpha$ expression was required for the ability of AR to suppress wound healing. The above conclusion was further corroborated by bone marrow transplantation between TNF-R1KO and GARKO mice. We found that GARKO bone marrow failed to accelerate wound healing in the $\gamma$-irradiated TNF-R1KO recipient mice (Figure 4E) compared with WT bone marrow. This is in contrast to the experiment shown in Figure 2B, in which GARKO bone marrow accelerated wound healing in WT recipient mice.

Taken together, the results shown in Figure 4, A-E, suggest that AR suppresses wound healing by enhancing local TNF- $\alpha$ expression.

AR enhances local TNF- $\alpha$ expression by promoting monocyte infiltration into wounds. We have shown that monocyte/macrophage $\mathrm{AR}$ is important for enhancing TNF- $\alpha$ expression in wound tissues. However, this could be due to an increase in infiltrating macrophages in the wound area and/or increased TNF- $\alpha$ production from macrophages. To test the possibility that AR promotes macrophage infiltration into wounds, we first used immunohistochemistry to detect macrophage populations and found fewer infiltrating macrophages in day 3 GARKO wounds compared with WT wounds (Figure 5, A and B).

We then asked whether AR regulated monocyte recruitment from peripheral blood. Flow cytometric analysis revealed that the PBMCs of GARKO mice contained a lower percentage of 

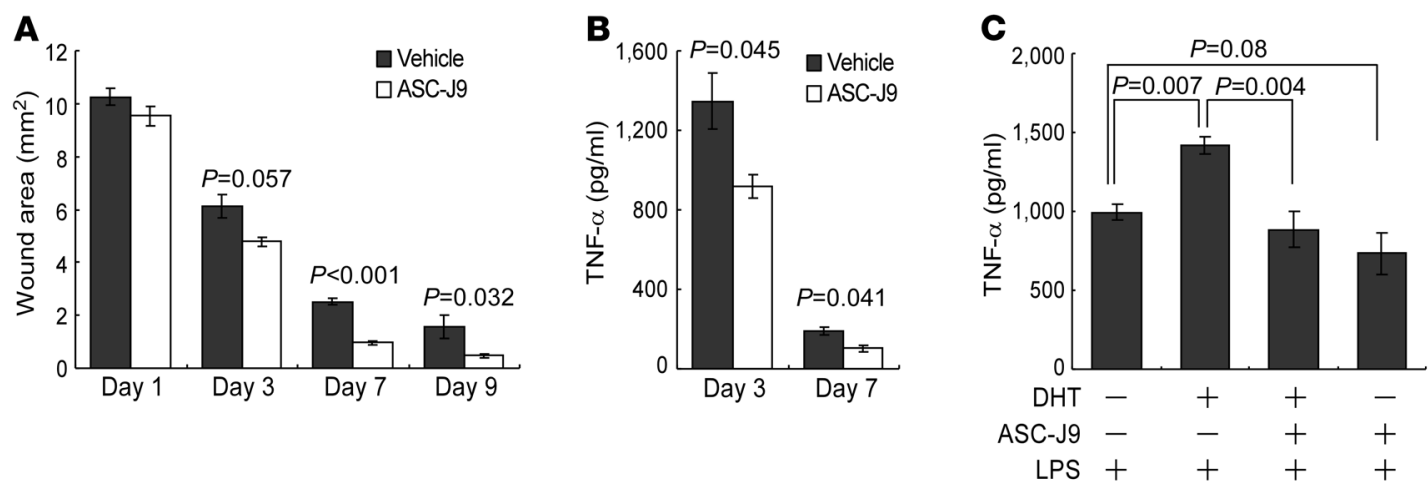

\section{Figure 6}

ASC-J9 accelerates cutaneous wound healing in WT mice and suppresses TNF- $\alpha$ production of wounds and BMMacs. (A) ASC-J9 cream $(50 \mu \mathrm{M})$ was applied topically on the wounds of WT mice daily, from day 0 to day 8 . The wound areas were quantified to compare the effects of ASC-J9 versus the vehicle cream. $n=3-4$ (6 wounds/mouse). (B) TNF- $\alpha$ protein expression was detected by ELISA in day 3 and day 7 wound tissues from ASC-J9- or vehicle-treated mice (pool 6 wounds/mouse in $0.7 \mathrm{ml}$ homogenization buffer). $n=4$. (C) Day 6 BMMacs $\left(8 \times 10^{4} /\right.$ well) were cultured in RPMI-CD medium overnight, then treated with $1 \mu \mathrm{M}$ ASC-J9 or DMSO for the entire culture period. Four hours after starting ASC-J9 treatment, $1 \mathrm{nM} \mathrm{DHT}$ or ethanol was added into the culture for another 4 hours, followed by $1 \mathrm{ng} / \mathrm{ml}$ LPS plus $1 \mu \mathrm{M}$ ASC-J9 or DMSO for 24 hours. TNF- $\alpha$ production in the supernatant was measured by ELISA.

$\mathrm{CD} 11 \mathrm{~b}^{+} \mathrm{F} 4 / 80^{+}$monocytes than WT mice. Interestingly, GARKO mice had a striking reduction in the inflammatory monocyte population $\left(\mathrm{CD} 11 \mathrm{~b}^{+} \mathrm{F} 4 / 80^{+} \mathrm{Gr} 1^{+}\right)$, which is known to be recruited into inflammatory tissues (33). In contrast, percentages of the resident monocyte subset $\left(\mathrm{CD} 11 \mathrm{~b}^{+} \mathrm{F} 4 / 80^{+} \mathrm{Gr} 1^{-}\right)$in PBMCs was similar between WT and GARKO mice (Figure 5C), suggesting that AR specifically increases the inflammatory monocyte pool in the peripheral blood, which is then recruited to the wound area.

Another possible mechanism by which AR might increase infiltrating macrophages in the wound areas could be through promoting monocyte chemotaxis. We found that the mRNA expression of Ccr2, a key chemokine receptor (34), was decreased in the bone marrow-derived monocytes/macrophages (BMMacs) from GARKO mice compared with WT mice (Figure 5D), suggesting that AR may be able to increase Cor2 gene expression. Using a luciferase reporter containing the putative androgen responsive element from the 5 '-promoter region of the mouse Ccr2 gene (Supplemental Figure 4A), we further found that AR, in the presence of DHT, could induce CCR2 promoter activity (Figure 5E). We then tested whether the increased CCR2 expression in monocytes/macrophages might result in promoting a chemotactic response to its ligand, MCP-1 (34), and the results showed that higher expression of CCR2 in WT BMMacs did increase their chemotaxis compared with the ARKO cells (Figure 5, F and G).

Taken together, the results from Figure 5, A-G, suggest that AR enhances macrophage infiltration into wounds by increasing the inflammatory monocyte pool in the peripheral blood and by augmenting monocyte recruitment into wound areas by increasing CCR2 expression on monocytes.

$A R$ induces TNF- $\alpha$ expression at the transcriptional level in macrophages. We also asked whether AR could directly enhance TNF- $\alpha$ expression in macrophages. First, we found that LPS-stimulated ARKO BMMacs had decreased expression of TNF- $\alpha$ compared with WT BMMacs (Figure 5H). Using a luciferase reporter containing the putative androgen responsive element found in the 5 -UTR $(-1,203$ to +122$)$ of the murine Tnfa gene (Supplemental Figure $4 \mathrm{~B}$ ), we found that $\mathrm{AR}$, in the presence of DHT, induced TNF- $\alpha$ promoter activity (Figure 5I).
Taken together, results from Figure 5, A-I, suggest that AR enhances TNF- $\alpha$ expression in the wound tissues by (a) increasing the circulating inflammatory monocyte population and promoting their recruitment into wound tissues, leading to an increase in macrophage infiltration into the wound area and (b) enhancing TNF- $\alpha$ expression at the transcriptional level in macrophages.

Therapeutic approach using ASC-J9 to target AR can accelerate wound healing. Using cell-specific ARKO mice and reciprocal bone marrow transplantation (Figures 1-5), we have demonstrated that AR can function through both androgen-dependent and -independent pathways to suppress wound healing and that the suppressive role of androgens in wound healing is exclusively mediated by AR. Therefore, AR (rather than androgens) plays a more central role in suppressing wound healing, and we conclude that targeting AR, and not androgens, may be a more promising therapy for wound healing. ASC-J9 is a newly developed anti-AR compound derived from curcumin that disrupts the interaction between $A R$ and $A R$ coregulators, resulting in increased AR degradation and decreased AR transactivation (35). Early therapeutic studies using mouse models and human cell lines have demonstrated that ASC-J9 can efficiently reduce AR-promoted tumor growth in liver (36) and bladder cancer (18), as well as AR-mediated spinal and bulbar muscular atrophy (SBMA or Kennedy disease), with little influence on serum testosterone concentrations $(35,36)$. We found that topical ASC-J9 treatment on WT mice resulted in accelerated wound healing (Figure 6A) and dampened local TNF- $\alpha$ expression (Figure 6B) compared with a control cream treatment. ASC-J9 also effectively reversed DHT-enhanced TNF- $\alpha$ expression in WT BMMacs (Figure 6C). Taken together, results shown in Figure 6 suggest that by inhibiting AR function, ASC-J9 thereby suppresses local TNF- $\alpha$ expression and consequently accelerates wound healing. Therefore, ASC-J9 may become a potential new therapeutic treatment for wound healing.

\section{Discussion}

Much remains unclear on the mechanisms by which androgens/AR suppress wound healing, and in particular, how different cell types utilize AR to regulate wound healing $(1,8)$. Here, we applied mul- 


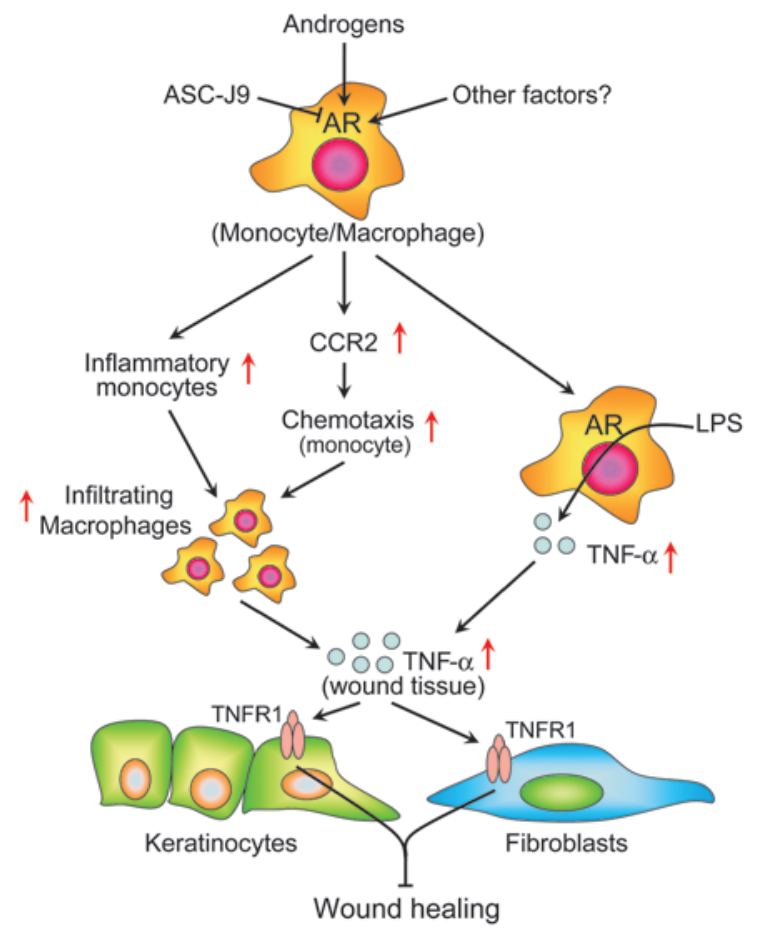

tiple approaches, including reciprocal bone marrow transplantation and several mouse strains with AR deficiency in specific cell types, to demonstrate that AR in monocytes/macrophages is critical in suppressing wound healing through enhancing local TNF- $\alpha$ production. Even though AR in keratinocytes and fibroblasts is dispensable for suppressing overall wound healing, AR in these 2 cell types plays key but opposing roles to regulate re-epithelialization. Furthermore, using in vivo and in vitro approaches, we demonstrated that monocyte/macrophage AR utilizes the following mechanisms to augment local TNF- $\alpha$ production: (a) increases circulating inflammatory monocyte population, (b) enhances monocyte recruitment and chemotaxis through upregulation of CCR2 expression, and (c) increases TNF- $\alpha$ expression at the transcriptional level in macrophages (Figure 7). The fact that AR can modulate the inflammatory response to regulate physiological activities makes it of great interest to investigate whether inflammatory diseases exhibiting sexual dimorphism, such as atherosclerosis $(37,38)$ and asthma (39), can also be modulated by AR in monocytes/macrophages. The further studies of AR function (in terms of inflammatory regulation) in these diseases will allow us to develop potential therapeutic approaches via targeting AR to combat such disorders.

Inflammatory mediators, especially TNF- $\alpha$, secreted by macrophages potently modulate the function of dermal fibroblasts and epithelial cells, thus making the macrophage a central regulator of the healing process $(31,40,41)$. Previous studies exploring the effects of TNF- $\alpha$ on the activity of resident skin cells have shown that TNF- $\alpha$ can inhibit type I collagen expression in wounds (30, $42,43)$ and counteract TGF- $\beta 1$-induced myofibroblast differentiation and collagen expression from dermal fibroblasts (31), thus indicating how augmented local TNF- $\alpha$ suppresses cutaneous wound healing. The ability of TNF- $\alpha$ to suppress the differentiation and $\alpha$-SMA expression of myofibroblasts (31), which appear later in the healing process and are important for wound

\section{Figure 7}

AR enhances local TNF- $\alpha$ expression from macrophages through multiple mechanisms to suppress cutaneous wound healing. Monocyte/ macrophage AR function can be modulated by androgens or other factors to enhance inflammatory responses induced by injury. Overall, local TNF- $\alpha$ expression from macrophages is augmented by AR and critically mediates wound-healing suppression by targeting resident skin cells (keratinocytes and/or fibroblasts) via TNF-R1. AR enhances local TNF- $\alpha$ expression through 3 mechanisms. First, AR increases the circulating inflammatory monocyte population. Second, AR promotes the expression of CCR2 on inflammatory monocytes to enhance their chemotaxis in response to MCP-1. Third, AR can directly enhance TNF- $\alpha$ expression of macrophages in response to LPS stimulation. Treatment with ASC-J9 can antagonize the function of AR and promote cutaneous wound healing.

contracture (44), might explain why we only observed significant differences in healing rates of WT and GARKO mice at the later stages, thus highlighting another critical way in which TNF- $\alpha$ mediates AR function in wound healing. In addition, the fact that local restoration of TNF- $\alpha$ can reverse accelerated wound healing in GARKO mice suggests that AR mainly affects local TNF- $\alpha$ production rather than the signals downstream of TNF receptor in the resident skin cells.

The rate of re-epithelialization (epithelium closure) usually links to the rate of wound closure (dermis closure) (27). However, our data suggest that these 2 processes can be uncoupled. For example, MARKO mice exhibited faster wound closure but a similar re-epithelialization rate compared with WT mice, while KARKO and FARKO mice showed delayed and enhanced re-epithelialization, respectively, but a comparable overall wound closure rate The delayed re-epithelialization observed in KARKO mice was not accompanied by a decrease in keratinocyte proliferation, suggesting that keratinocyte AR might promote re-epithelialization by enhancing keratinocyte migration. Such an implication contradicts a recent study by Gilliver et al. (10). In that study, DHT treatment inhibited keratinocyte migration in an in vitro migration assay (10), but the effect only became significant at a high dose $(100 \mathrm{nM})$, which is $1-2$ orders of magnitude higher than physiological levels of DHT (1-10 nM). Hence, the decrease in keratinocyte migration might have been caused by cytotoxicity rather than through physiological DHT signaling. In addition, their in vitro culture system does not account for possible cross-talk among different cell types involved in re-epithelialization. Our results using FARKO mice suggest that $\mathrm{AR}$ in fibroblasts, rather than in keratinocytes or myeloid cells, is important for suppressing re-epithelialization. Fibroblast AR might suppress re-epithelialization by inhibiting MCP-1 production. MCP-1 levels correlate well with re-epithelialization rates, as GARKO and FARKO mice had higher MCP-1 expression (Figure 4 and Supplemental Figure 5A) and more rapid re-epithelialization, whereas MARKO mice had MCP-1 levels (Supplemental Figure 3) and re-epithelialization rates comparable to those in WT mice. In line with this, re-epithelialization rate is decreased in MCP-1 knockout mice (45). These data suggest that MCP-1 levels might be critical in controlling re-epithelialization, although further studies will be necessary to clarify whether and how fibroblast AR regulates MCP-1 to promote re-epithelialization. In summary, AR seems to play a major role in fibroblasts by suppressing MCP-1 expression, which consequently delays re-epithelialization. In contrast, AR in keratinocytes enhances re-epithelialization by enhancing keratinocyte migration (Supplemental Figure 5B). 
The increased collagen content in GARKO wounds suggests that AR suppresses collagen deposition. However, in vitro treatment of dermal fibroblasts with androgens stimulated collagen production (9), and we observed similar collagen deposition in the healing wounds of FARKO and WT mice, suggesting that fibroblast AR does not repress collagen deposition. Rather, our data show that MARKO mice have increased collagen deposition, suggesting that macrophage AR is important for suppressing collagen deposition. The rate of collagen deposition is determined by the balance of collagen synthesis and degradation. Interestingly, the activity of MMP-1 (collagenase I) and MMP-13 (collagenase III) was reduced in castrated rats in the later stages of wound healing (9). Because macrophages express several proteinases and their activators/inhibitors (46-49), it is possible that macrophage AR promotes the expression or activation of proteinases to enhance collagen degradation. The increased TNF- $\alpha$ production from macrophages might also contribute to collagen degradation, as TNF- $\alpha$ has been shown to induce the expression of several proteinases, including MMP-1, from fibroblasts, while suppressing the secretion of MMP-1 inhibitors $(29,50)$. In addition, TNF- $\alpha$ can counteract TGF- $\beta 1$-induced collagen synthesis from fibroblasts $(31,51)$, providing another possible mechanism by which AR might repress collagen deposition (Supplemental Figure 6). Wounds of GARKO mice showed increased collagen deposition, which usually promotes wound closure and increases the tensile strength of regenerated tissues $(44,52)$. However, excess collagen deposition is not necessarily beneficial for healing and may lead to the development of hypertrophic scars, keloids, or severe fibrosis $(44,53,54)$. Further studies are necessary to clarify the role of $\mathrm{AR}$ in scar formation, especially in settings where AR is targeted for wound-healing therapy.

Heterogeneity in monocyte/macrophage populations has been demonstrated in both humans and mice $(33,55,56)$. Nahrendorf and colleagues recently reported (55) that 2 subsets of monocytes are involved in the healing myocardium. In the early phase, inflammatory monocytes (Ly-6C ${ }^{\text {hi }} \mathrm{Gr} 1{ }^{\text {hi }} \mathrm{CCR} 2{ }^{+} \mathrm{CXCR} 1^{\mathrm{lo}}$ ) are predominantly recruited and play a major role in phagocytosis and clearance of infection. In the later phase, resident monocytes (Ly-6C ${ }^{\text {lo }} \mathrm{Gr} 1^{\text {lo } C C R 2-C X C R} 1^{\text {hi }}$ ) are recruited and play roles in repair and angiogenesis (55). Based on our data, we believe that AR mainly influences the functions of inflammatory monocytes rather than the resident monocytes and plays a more critical role in the inflammatory phase of wound healing.

CCR2 has been linked to the recruitment of circulating monocytes into inflamed tissues $(57,58)$, as well as emigration of monocytes from the bone marrow into the circulation (59). In our studies, we found that the circulating inflammatory monocyte population was reduced in the blood of GARKO mice. This could be due to a reduced monocyte population in the bone marrow and/or reduced CCR2 expression on monocytes. Interestingly, our studies showed that the monocyte population $\left(\mathrm{CD} 11 \mathrm{~b}^{+} \mathrm{F} 4 / 80^{+}\right)$is reduced in GARKO compared with WT bone marrow (Supplemental Figure 7). Further studies are necessary to clarify the role of AR in the development of monocytes in the bone marrow.

Earlier studies have suggested that castration or systemic blockade of androgens might accelerate wound healing (1) and represent a potential approach to wound-healing therapy in elderly males. However, from a therapeutic point of view, these approaches have enormous side effects, such as impotence, loss of libido, osteoporosis, and fatigue $(35,60)$, which need to be carefully considered by urologists before being used as therapies to treat prostate cancer patients. Castration and androgen blocking are therefore very unlikely to be used as therapies to treat wound healing. On the other hand, the use of ASC-J9 to target AR instead of androgens leads to a loss of AR function with limited influence on serum testosterone levels, sexual function, or fertility (35). As a topical treatment using ASC-J9 to treat acne vulgaris, phase I and phase II clinical studies conducted by AndroScience Corp. have shown no severe adverse effect (C. Shih, personal communication), and our results indicate that it is feasible to suppress AR function by using ASC-J9 as a topical treatment to hasten wound healing (Figure 6A).

Importantly, we demonstrate in vivo evidence that androgen effects are not equal to AR effects. Early in vitro studies using cell lines showed that androgens could signal through AR-independent pathways to regulate cellular activities $(11,17,61)$; therefore, using castration or antiandrogens to decrease androgen levels do not address whether the ability of androgens to inhibit wound healing is mediated by AR-dependent or -independent pathways. Using GARKO mice with DHT restoration, we showed that ARdependent pathways, rather than AR-independent pathways, are critical in mediating the ability of androgens to suppress wound healing (Figure 1H). On the other hand, AR can be activated by other factors in addition to androgens, such as estrogens (62, $63)$, antiandrogens $(64,65)$, and kinases $(66,67)$. Interestingly, our recent studies suggested that the androgen-independent activity of AR is important for promoting neutrophil differentiation (14). In line with this, our data also demonstrate that AR of bone marrow cells can suppress wound healing even when there is low testosterone in the host, supporting the notion that AR can function in an androgen-independent manner. Overall, our study suggests that androgens operate exclusively through AR to suppress wound healing, while AR can function though both androgen-dependent and androgen-independent pathways to suppress wound healing. Therefore, we believe that AR, rather than androgens, has a more central role in wound-healing suppression, which is difficult to verify in castration- or antiandrogen-flutamide-treated models. Therefore, AR represents a more attractive therapeutic target than do androgens for accelerating wound healing. Further studies on ASC-J9 and similar drugs are needed to investigate how AR can be selectively targeted in specific cell types while limiting effects on serum androgen levels. Such studies will facilitate the development of more selective drugs to battle androgen/AR-dependent diseases.

\section{Methods}

Mice, cells, and reagents. fAR mice on a C57BL/ 6 background were generated by inserting loxP sites to flank exon 2 of Argene (15). C57BL/6, ACTBCre (FVB background), LyzCre (C57BL/6 background), and TNF-R1KO (C57BL/6 background) mice were purchased from The Jackson Laboratory. Fsp1Cre (C57BL/6 background) mice were a gift from N.A. Bhowmick (Vanderbilt University, Nashville, Tennessee, USA; ref. 26), and K5Cre (FVB background) mice were a gift from D.G. Johnson (University of Texas MD Anderson Cancer Center, Smithville, Texas, USA) (25). Animal protocols and usage were approved by the University of Rochester Committee on Animal Resources, and the mice were kept in a specific pathogen-free environment at the animal facility of the University of Rochester. pWPI-mAR was generated by inserting the full-length mouse Ar gene into pWPI vectors. pGL3-TNF $(-1,203$ to +2$)$ was a gift from J.S. Schorey (University of Notre Dame, Notre Dame, Indiana, USA; 
ref. 68). pGL3-TNF $(-1,203$ to +122$)$ was generated by inserting the $-1,203$ to +122 promoter region of the murine Tnfa gene into pGL3-Luc basic vector. pGL3-mCCR 2 was generated by inserting the $-2,058$ to +27 promoter region of the murine Ccr2 gene into pGL3-Luc basic vector. ASC-J9 and vehicle cream were gifts from AndroScience Corp., which developed and prepared the cream.

Creation of cutaneous wounds and DHT restoration. To create the excisional wounds, 8- to 12-week-old male mice were anesthetized and the dorsal skin was shaved. After sanitizing with $70 \%$ ethanol, 2 or 6 full-thickness wounds were created on the dorsal skin using 4-mm-diameter dermal biopsy punches (Miltex Inc.). Wound closure was temporally monitored by planimetric measurement, in which photographs of each wound taken at various time points were analyzed by Image (NIH) to calculate the wound areas using the edges of dermis as the wound edges. In DHT restoration experiments, DHT $(5 \mathrm{mg} /$ pellet, 60-day release; Innovative Research of America) or placebo pellets were subcutaneously implanted into WT and GARKO mice 2 weeks before creating wounds.

Histology and immunohistochemistry analysis. To determine re-epithelialization rates, day 3 wound tissues were excised from mice, fixed in $10 \%$ neutralized formaldehyde for 24 hours, and subjected to paraffin section at $5 \mu \mathrm{m}$ thickness and subsequent H\&E staining. Photographs of the wound sections were used to measure the width between 2 epithelial edges and the width between 2 wound edges (dermis edges) by ImageJ software. To measure re-epithelialization (epithelium closure), we calculated the percentage of wound width (distance between the opposing dermis edges) covered by epithelium, using the following formula: re-epithelialization $=([$ distance between opposing dermis edges - distance between opposing epithelium edges]/distance between opposing dermis edges) $\times 100 \%$.

To detect proliferating epithelial cells in wound sections, day 3 wounds were harvested and subjected to paraffin sectioning. Two hours before harvest, $\mathrm{BrdU}(150 \mu \mathrm{g} / \mathrm{g}$ body weight) was intraperitoneally injected into each mouse. Sections of $5 \mu \mathrm{m}$ were collected and subjected to Zymed BrdU Staining Kit (Invitrogen) according to the manufacturer's manual. The total number of proliferating $\left(\mathrm{BrdU}^{+}\right)$epithelial cells in the wound area of each wound section was counted. To detect collagen deposition, $10-\mu \mathrm{m}$ paraffin sections of wounds were subjected to Masson's Trichrome Staining (Sigma-Aldrich) according to the manufacturer's manual. Using this method, collagen fibers stained blue, nuclei stained black, and cytoplasm and muscle fibers stained red. To quantify collagen deposition, microscopic photos of trichrome staining sections were analyzed by ImageJ software to compare the relative blue color intensity in granulation tissues and the adjacent dermis. To detect the infiltrating macrophages and myeloid cells, day 3 wounds were excised, fixed in $4 \%$ paraformaldehyde for 30 minutes, and subjected to cryosection. Sections of $5 \mu \mathrm{m}$ were subjected to immunohistochemistry with anti-F4/80 and anti-CD11b primary antibodies (eBioscience). After washing, the samples were incubated with biotinylated secondary antibodies, followed by incubation with avidin-HRP solution. The samples were then developed with DAB solution $\left(0.06 \% \mathrm{DAB}\right.$ in $0.05 \mathrm{M}$ Tris/pH 7.6 with $\left.0.03 \% \mathrm{H}_{2} \mathrm{O}_{2}\right)$ and counterstained with hematoxylin. Five photographs were randomly taken under $\times 400$ magnification in each wound area, and the number of cells with positive staining was counted per area.

Bone marrow transplantation. Six-week-old mice were $\gamma$-irradiated (10-12 Gy) and adoptively transferred with donor bone marrow cells $\left(5 \times 10^{6} /\right.$ mice) 24 hours after irradiation. Donor bone marrow cells were given 6 weeks to repopulate, and mice were treated with antibiotic water containing $1.1 \mathrm{mg} / \mathrm{ml}$ neomycin and $850 \mathrm{U} / \mathrm{ml}$ polymyxin-B sulfate before wounds were created on their skin. At the end point of wound-healing experiments, the spleen, thymus, bone marrow cells, wound tissues, and non-wounded skin were harvested for genomic DNA extraction. PCR was used to deter- mine the genotype of AR alleles in each tissue, as previously described, to confirm the success of bone marrow transplantation (15).

RT-PCR to detect the expression of inflammatory mediators in wound tissues. Non-wounded skin and day 3 wound tissues were excised by $6-\mathrm{mm}$ diameter dermal biopsy punches and homogenized in $1 \mathrm{ml}$ Trizol reagent (Invitrogen). Total RNA extraction was performed according to the manufacturer's manual. RNA $(5 \mu \mathrm{g})$ was used for reverse transcription using SuperScript III Reverse Transcriptase (Invitrogen). The cDNA was subjected to real-time PCR to detect mRNA level of Tnfa, Mcp1, Mip1, $T g f b 1$, Inos, Il1b, and E-selectin using SYBR GreenER (Invitrogen) and iCycler system (BioRad).

ELISA to detect inflammatory mediators in the wound tissues. To detect cytokine levels in wound tissues, 2 or 4 wounds from each mouse were excised with 6 - $\mathrm{mm}$ biopsy punches and homogenized in $0.7 \mathrm{ml}$ homogenization buffer ( $1 \times$ PBS, $0.1 \%$ SDS, $1 \%$ Tween-20, 5 mM EDTA) containing protease inhibitor (Roche Diagnostics). Non-wounded skin samples ( 2 or 4 pieces for each mice) excised by $6-\mathrm{mm}$ punches served as controls. The homogenized samples were centrifuged at 20,817 $\mathrm{g}$ for 10 minutes at $4{ }^{\circ} \mathrm{C}$, and the supernatant was transferred to a fresh tube to detect concentrations of TNF- $\alpha$, MCP-1, IL- $1 \beta$, IFN- $\gamma$, IL- 6 , and the active form of TGF- $\beta 1$ using the ELISA kit (eBioscience) according to the manufacturer's manual. To detect total TGF- $\beta 1$ concentration, $100 \mu \mathrm{l}$ of the samples were first treated with $20 \mu \mathrm{l} 1 \mathrm{~N} \mathrm{HCl}$ for 20 minutes, followed by addition of $20 \mu \mathrm{l} 1 \mathrm{~N} \mathrm{NaOH}$ to neutralize the samples. Final concentrations were adjusted by the dilution factor of 1.4 .

Analysis of monocyte populations in PBMCs. Blood (1 ml) was drawn from the left ventricle of each mouse with a heparin-rinsed syringe and diluted with $1 \mathrm{ml}$ PBS. The diluted blood was carefully overlaid onto $3 \mathrm{ml}$ Histopaque 1077 (Sigma-Aldrich) and centrifuged at $800 \mathrm{~g}$ at room temperature for 20 minutes without brake. Cells at the interface of the serum and histopaque were carefully collected, washed twice with medium, and subjected to staining for flow cytometry analysis. The antibodies used to identify monocyte populations were anti-CD11b-APC, anti-F4/80-PECy5, anti-Gr1-PE, and anti-CD45-FITC (eBioscience). CD 45 ${ }^{+}$cells were gated for monocyte population analysis.

Local TNF- $\alpha$ restoration and ASC-J9 treatment. In TNF- $\alpha$ restoration experiments, $50 \mu$ l of recombinant TNF- $\alpha$ (R\&D Systems) was intradermally injected at various concentrations around each GARKO wound 24 hours after creating the wounds. Injection of $50 \mu \mathrm{l}$ PBS around the wounds served as controls. For the ASC-J9 treatment, topical application of ASC-J9 $(50 \mu \mathrm{M})$ or control cream was applied daily to the wounds of WT C57BL/6 male mice.

Culture of BMMacs for chemotaxis assay and TNF- $\alpha$ ELISA. Bone marrow cells were harvested from femurs and tibias of mice, and $10^{7}$ cells were cultured in a $15-\mathrm{cm}$ petri dish with $25 \mathrm{ml}$ RPMI medium containing $20 \mathrm{ng} / \mathrm{ml}$ M-CSF. BMMacs were harvested on day 3 for chemotaxis assays. Alternatively, an additional $25 \mathrm{ml}$ RPMI medium supplemented with $20 \mathrm{ng} / \mathrm{ml}$ M-CSF were added to bone marrow cells on day 4, and BMMacs were harvested on day 6 for assessment of TNF- $\alpha$ expression. For chemotaxis assays, $1.5 \times 10^{5}$ day 3 BMMacs were seeded onto transwell inserts $(8-\mu \mathrm{m}$ pore size; Corning Inc.) in $200 \mu \mathrm{l}$ serum-free RPMI medium. The lower chamber contained $600 \mu \mathrm{l}$ RPMI- $0.5 \%$ charcoal/dextran-treated FCS (RPMI-5\% CD) medium with $200 \mathrm{ng} / \mathrm{ml}$ MCP-1 (R\&D Systems). Cells were incubated for 2 hours at $37^{\circ} \mathrm{C}$, fixed with $4 \%$ paraformaldehyde, and stained with $\mathrm{H} \& \mathrm{E}$, and cells that had crossed the membrane were counted. For measuring TNF- $\alpha$ expression, $5 \times 10^{4}$ day 6 BMMacs were seeded into 96-well plates in $200 \mu \mathrm{l}$ RPMI medium overnight and treated with LPS for another 24 hours, and culture supernatants were collected for TNF- $\alpha$ ELISA.

Luciferase assay. NIH3T3 cells were cultured in RPMI-10\% CD medium and co-transfected overnight with pGL3 reporter plasmids and PWPI-AR or pWPI vector using Lipofectamine 2000 (Invitrogen) according to the 
manufacturer's manual. After treating with $10 \mathrm{nM} \mathrm{DHT}$ or ethanol for 24 hours, luciferase activity was measured by Dual-Luciferase Assay (Promega) according to the manufacturer's manual.

Statistics. The data are presented as mean \pm SEM unless specified. For parametric analyses, 2-tailed Student's $t$ test or 1-way ANOVA were used. For nonparametric analyses, Mann-Whitney $U$ test or Kruskal-Wallis test were used. A $P$ value less than 0.05 was considered statistically significant.

\section{Acknowledgments}

We thank Karen Wolf and Janice Chen for manuscript preparation and Therese Wiedmer and Patricia J. Simpson-Haidaris for criti- cal review. We also thank Charles Shih (AndroScience Corp., San Diego, California, USA) for technical assistance. This work was supported by NIH grant DK73414 and a George Whipple Professorship Endowment.

Received for publication March 26, 2009, and accepted in revised form September 16, 2009.

Address correspondence to: Chawnshang Chang, Box 626, 601 Elmwood Avenue, Rochester, New York 14642, USA. Phone:(585) 273-4500; Fax: (585) 756-4133; E-mail: chang@URMC.rochester.edu.
1. Ashcroft, G.S., and Mills, S.J. 2002. Androgen receptor-mediated inhibition of cutaneous wound healing. J. Clin. Invest. 110:615-624.

2. Ashcroft, G.S., Greenwell-Wild, T., Horan, M.A., Wahl, S.M., and Ferguson, M.W. 1999. Topical estrogen accelerates cutaneous wound healing in aged humans associated with an altered inflammatory response. Am. J. Pathol. 155:1137-1146.

3. Fimmel, S., and Zouboulis, C.C. 2005. Influence of physiological androgen levels on wound healing and immune status in men. Aging Male. 8:166-174.

4. Taylor, R.J., Taylor, A.D., and Smyth, J.V. 2002. Using an artificial neural network to predict healing times and risk factors for venous leg ulcers. J. Wound Care. 11:101-105.

5. Chen, W., et al. 1998. Evidence of heterogeneity and quantitative differences of the type 15 alphareductase expression in cultured human skin cells - evidence of its presence in melanocytes. J. Invest. Dermatol. 110:84-89.

6. Chang, C.S., Kokontis, J., and Liao, S.T. 1988. Molecular cloning of human and rat complementary DNA encoding androgen receptors. Science. 240:324-326.

7. Heinlein, C.A., and Chang, C. 2004. Androgen receptor in prostate cancer. Endocr. Rev. 25:276-308.

8. Gilliver, S.C., Ashworth, J.J., Mills, S.J., Hardman, M.J., and Ashcroft, G.S. 2006. Androgens modulate the inflammatory response during acute wound healing. J. Cell Sci. 119:722-732.

9. Gilliver, S.C., Ruckshanthi, J.P., Atkinson, S.J., and Ashcroft, G.S. 2007. Androgens influence expression of matrix proteins and proteolytic factors during cutaneous wound healing. Lab. Invest. 87:871-881.

10. Gilliver, S.C., Ruckshanthi, J.P., Hardman, M.J., Zeef, L.A., and Ashcroft, G.S. 2009. 5alpha-dihydrotestosterone (DHT) retards wound closure by inhibiting re-epithelialization. J. Pathol. 217:73-82.

11. Rahman, F., and Christian, H.C. 2007. Non-classical actions of testosterone: an update. Trends Endocrinol. Metab. 18:371-378.

12. Cao, X., et al. 2006. Regulator of G-protein signaling 2 (RGS2) inhibits androgen-independent activation of androgen receptor in prostate cancer cells. Oncogene. 25:3719-3734.

13. Kasbohm, E.A., et al. 2005. Androgen receptor activation by $\mathrm{G}(\mathrm{s})$ signaling in prostate cancer cells. J. Biol. Chem. 280:11583-11589.

14. Chuang, K.H., et al. 2009. Neutropenia with impaired host defense against microbial infection in mice lacking androgen receptor. J. Exp. Med. 206:1181-1199.

15. Yeh, S., et al. 2002. Generation and characterization of androgen receptor knockout (ARKO) mice: an in vivo model for the study of androgen functions in selective tissues. Proc. Natl. Acad. Sci. U. S. A. 99:13498-13503.

16. Braiman-Wiksman, L., Solomonik, I., Spira, R., and Tennenbaum, T. 2007. Novel insights into wound healing sequence of events. Toxicol. Pathol. 35:767-779.

17. Benten, W.P., et al. 1999. Testosterone signaling through internalizable surface receptors in andro- gen receptor-free macrophages. Mol. Biol. Cell. 10:3113-3123

18. Miyamoto, H., et al. 2007. Promotion of bladder cancer development and progression by androgen receptor signals. J. Natl. Cancer Inst. 99:558-568.

19. Martin, P., and Leibovich, S.J. 2005. Inflammatory cells during wound repair: the good, the bad and the ugly. Trends Cell Biol. 15:599-607.

20. DiPietro, L.A. 1995. Wound healing: the role of the macrophage and other immune cells. Shock. 4:233-240.

21. Leibovich, S.J., and Ross, R. 1975. The role of the macrophage in wound repair. A study with hydrocortisone and antimacrophage serum. Am. J. Pathol. 78:71-100.

22. Clausen, B.E., Burkhardt, C., Reith, W., Renkawitz, R., and Forster, I. 1999. Conditional gene targeting in macrophages and granulocytes using LysMcre mice. Transgenic Res. 8:265-277.

23. Dovi, J.V., He, L.K., and DiPietro, L.A. 2003. Accelerated wound closure in neutrophil-depleted mice. J. Lenkoc. Biol. 73:448-455.

24. Simpson, D.M., and Ross, R. 1972. The neutrophilic leukocyte in wound repair a study with antineutrophil serum. J. Clin. Invest. 51:2009-2023.

25. Berton, T.R., et al. 2003. Tumor formation in mice with conditional inactivation of Brca1 in epithelial tissues. Oncogene. 22:5415-5426.

26. Bhowmick, N.A., et al. 2004. TGF-beta signaling in fibroblasts modulates the oncogenic potential of adjacent epithelia. Science. 303:848-851.

27. Reynolds, L.E., et al. 2005. Accelerated re-epithelialization in beta3-integrin-deficient- mice is associated with enhanced TGF-beta1 signaling. Nat. Med. 11:167-174.

28. Hebda, P.A. 1988. Stimulatory effects of transforming growth factor-beta and epidermal growth factor on epidermal cell outgrowth from porcine skin explant cultures. J. Invest. Dermatol. 91:440-445.

29. Eming, S.A., Krieg, T., and Davidson, J.M. 2007. Inflammation in wound repair: molecular and cellular mechanisms. J. Invest. Dermatol. 127:514-525.

30. Buck, M., Houglum, K., and Chojkier, M. 1996. Tumor necrosis factor-alpha inhibits collagen alpha1(I) gene expression and wound healing in a murine model of cachexia. Am. J. Pathol. 149:195-204.

31. Goldberg, M.T., et al. 2007. TNF-alpha suppresses alpha-smooth muscle actin expression in human dermal fibroblasts: an implication for abnormal wound healing. J. Invest. Dermatol. 127:2645-2655.

32. Mori, R., Kondo, T., Ohshima, T., Ishida, Y., and Mukaida, N. 2002. Accelerated wound healing in tumor necrosis factor receptor p55-deficient mice with reduced leukocyte infiltration. FASEBJ. 16:963-974.

33. Geissmann, F., Jung, S., and Littman, D.R. 2003. Blood monocytes consist of two principal subsets with distinct migratory properties. Immunity. 19:71-82.

34. Mantovani, A., et al. 2004. The chemokine system in diverse forms of macrophage activation and polarization. Trends Immunol. 25:677-686.
35. Yang, Z., et al. 2007. ASC-J9 ameliorates spinal and bulbar muscular atrophy phenotype via degradation of androgen receptor. Nat. Med. 13:348-353.

36. Ma, W.L., et al. 2008. Androgen receptor is a new potential therapeutic target for the treatment of hepatocellular carcinoma. Gastroenterology. 135:947-955, 955.e1-955.e5.

37. McGrath, K.C., McRobb, L.S., and Heather, A.K. 2008. Androgen therapy and atherosclerotic cardiovascular disease. Vasc. Health Risk Manag. 4:11-21.

38. Liu, P.Y., Death, A.K., and Handelsman, D.J. 2003. Androgens and cardiovascular disease. Endocr. Rev. 24:313-340.

39. Pergola, C., et al. 2008. ERK-mediated regulation of leukotriene biosynthesis by androgens: a molecular basis for gender differences in inflammation and asthma. Proc. Natl. Acad. Sci. U. S. A. 105:19881-19886.

40. Rappolee, D.A., Mark, D., Banda, M.J., and Werb, Z. 1988. Wound macrophages express TGF-alpha and other growth factors in vivo: analysis by mRNA phenotyping. Science. 241:708-712.

41. Riches, D.H.W. 1996. Macrophage involvement in wound repair, remodeling, and fibrosis. In The molecular and cellular biology of wound repair. R.A.F. Clark, editor. Plenum Press. New York, New York, USA. 95-141.

42. Salomon, G.D., et al. 1991. The local effects of cachectin/tumor necrosis factor on wound healing. Ann. Surg. 214:175-180.

43. Rapala, K., et al. 1991. Tumor necrosis factor alpha inhibits wound healing in the rat. Eur. Surg. Res. 23:261-268.

44. Singer, A.J., and Clark, R.A. 1999. Cutaneous wound healing. N. Engl. J. Med. 341:738-746.

45. Low, Q.E., et al. 2001. Wound healing in MIP1alpha(-/-) and MCP-1(-/-) mice. Am. J. Pathol. 159:457-463.

46. Xiong, W., et al. 2009. Membrane-type 1 matrix metalloproteinase regulates macrophage-dependent elastolytic activity and aneurysm formation in vivo. J. Biol. Chem. 284:1765-1771.

47. Schneider, F., et al. 2008. Matrix-metalloproteinase-14 deficiency in bone-marrow-derived cells promotes collagen accumulation in mouse atherosclerotic plaques. Circulation. 117:931-939.

48. Ohtani, H. 1998. Stromal reaction in cancer tissue: pathophysiologic significance of the expression of matrix-degrading enzymes in relation to matrix turnover and immune/inflammatory reactions. Pathol. Int. 48:1-9.

49. Visse, R., and Nagase, H. 2003. Matrix metalloproteinases and tissue inhibitors of metalloproteinases: structure, function, and biochemistry. Circ. Res. 92:827-839.

50. O'Kane, C.M., Elkington, P.T., and Friedland, J.S. 2008. Monocyte-dependent oncostatin M and TNF-alpha synergize to stimulate unopposed matrix metalloproteinase $-1 / 3$ secretion from human lung fibroblasts in tuberculosis. Eur. J. Immunol. 38:1321-1330.

51. Leask, A., and Abraham, D.J. 2004. TGF-beta signaling and the fibrotic response. FASEB J. 18:816-827. 
52. Bhowmick, N.A., et al. 2002. The conditional knockout of the transforming growth factor type II receptor in fibroblasts results in impaired wound healing [abstract]. Wound Repair and Regeneration. 10:A4

53. Stramer, B.M., Mori, R., and Martin, P. 2007. The inflammation-fibrosis link? A Jekyll and Hyde role for blood cells during wound repair. J. Invest. Dermatol. 127:1009-1017.

54. Ehrlich, H.P. 2000. Collagen considerations in scarring and regenerative repair. In Scarless wound healing. H.G. Garg and M.T. Longaker, editors. Marcel Dekker Inc. New York, New York, USA. 99-113.

55. Nahrendorf, M., et al. 2007. The healing myocardium sequentially mobilizes two monocyte subsets with divergent and complementary functions. J. Exp. Med. 204:3037-3047.

56. Gordon, S., and Taylor, P.R. 2005. Monocyte and macrophage heterogeneity. Nat. Rev. Immunol. 5:953-964.

57. Serbina, N.V., Salazar-Mather, T.P., Biron, C.A. Kuziel, W.A., and Pamer, E.G. 2003. TNF-/iNOSproducing dendritic cells mediate innate immune defense against bacterial infection. Immunity. 19:59-70.
58. Kurihara, T., Warr, G., Loy, J., and Bravo, R. 1997. Defects in macrophage recruitment and host defense in mice lacking the CCR 2 chemokine receptor. J. Exp. Med. 186:1757-1762.

59. Serbina, N.V., and Pamer, E.G. 2006. Monocyte emigration from bone marrow during bacterial infection requires signals mediated by chemokine receptor CCR2. Nat. Immunol. 7:311-317.

60. Berges, R., and Bello, U. 2006. Effect of a new leuprorelin formulation on testosterone levels in patients with advanced prostate cancer. Curr. Med. Res. Opin. 22:649-655.

61.Sun, Y.H., Gao, X., Tang, Y.J., Xu, C.L., and Wang, L.H. 2006. Androgens induce increases in intracellular calcium via a $\mathrm{G}$ protein-coupled receptor in LNCaP prostate cancer cells. J. Androl. 27:671-678.

62. Yeh, S., Miyamoto, H., Shima, H., and Chang, C. 1998. From estrogen to androgen receptor: a new pathway for sex hormones in prostate. Proc. Natl. Acad. Sci. U. S. A. 95:5527-5532.

63. Lanzino, M., et al. 2005. Endogenous coactivator ARA70 interacts with estrogen receptor alpha (ERalpha) and modulates the functional ERalpha/ androgen receptor interplay in MCF-7 cells. J. Biol. Chem. 280:20421-20430.

64. Yeh, S., Miyamoto, H., and Chang, C. 1997. Hydroxyflutamide may not always be a pure antiandrogen. Lancet. 349:852-853.

65. Miyamoto, H., Yeh, S., Wilding, G., and Chang, C. 1998. Promotion of agonist activity of antiandrogens by the androgen receptor coactivator, ARA70, in human prostate cancer DU145 cells. Proc. Natl. Acad. Sci. U. S. A. 95:7379-7384.

66. Gao, H., et al. 2006. Combinatorial activities of Akt and B-Raf/Erk signaling in a mouse model of androgen-independent prostate cancer. Proc. Natl. Acad. Sci. U. S. A. 103:14477-14482.

67. Guo, Z., et al. 2006. Regulation of androgen receptor activity by tyrosine phosphorylation. Cancer Cell. 10:309-319.

68. Lee, S.B., and Schorey, J.S. 2005. Activation and mitogen-activated protein kinase regulation of transcription factors Ets and NF-kappaB in Mycobacterium-infected macrophages and role of these factors in tumor necrosis factor alpha and nitric oxide synthase 2 promoter function. Infect. Immun. 73:6499-6507. 